\title{
Jeremias Gotthelfs Neuer Berner-Kalender und seine schweizerischen Konkurrenten
}

Die geringe Resonanz, welche die von Jeremias Gotthelf redigierten Jahrgänge des Neuen Berner-Kalenders in der germanistischen Forschung gefunden haben, ist ein Indiz für den Stellenwert, der seiner Kalendertätigkeit zugebilligt wurde und immer noch wird: Innerhalb des imposanten Werks des Emmentaler Dichters und Pfarrers, so die verbreitete Auffassung, kommt den für den Neuen Berner-Kalender verfaßten Beiträgen eine allenfalls marginale Bedeutung zu. Ganz anders sah dies offenbar Gotthelf selbst. Als ihm die Bernische gemeinnützige Gesellschaft Ende 1838 die Redaktion des von ihr initiierten Neuen Berner-Kalenders antrug, nahm er das Angebot ohne zu zögern an. Innerhalb weniger Tage entwarf er mehrere „Kalenderproben“, die er seinem Freund Carl Bitzius mit einem Brief übermittelte, der Gotthelfs Selbstverständnis als Kalenderautor erhellt.' Die in Gotthelfs Korrespondenz formulierten programmatischen Überlegungen, ${ }^{2}$ die Tatsache, daß Gotthelf, wie er in einem Brief vom 16. Dezember 1838 an Carl Bitzius verrät, bereits 1834 erfolglos versucht hatte, als Kalenderautor Fuß zu fassen, ${ }^{3}$ der Austausch mit Johann Jakob Reithard, der seit 1834 als Herausgeber des Republikaner-Kalenders fungierte, ${ }^{4}$ und nicht zuletzt die Schnelligkeit, mit der Gotthelf den ersten von ihm betreuten Jahrgang des Neuen Berner-Kalenders fertigstellte, ${ }^{5}$ machen deutlich, mit welch hohen Erwartungen und mit welchem Eifer Gotthelf ans Werk ging. Wenn der Emmentaler Pfarrer in der Folge nicht wenige seiner zuerst im Neuen Berner-Kalender erschienenen Erzählungen an anderer Stelle publizierte, dürfte dies nicht allein verlegerischen und ökonomischen Interessen geschuldet sein. Offenkundig hat er den Kalenderbeiträgen nicht weniger literarische Dignität zugesprochen als seinen anderen belletristischen Schriften. ${ }^{6}$ Die Redaktion des Neuen Berner-Kalenders verstand Gotthelf nie als eine von

1 Vgl. Silvia Serena Tschopp: Predigen, gefaßt in Lebenssprache. Zur narrativen Strategie von Gotthelfs Neuem Berner-Kalender. In: Erzählkunst und Volkserziehung. Das literarische Werk des Jeremias Gotthelf. Hrsg. von Walter Pape, Hellmut Thomke u. Silvia Serena Tschopp. Tübingen 1999, S. 111 127, hier S. 111.

2 Vgl. dazu den immer noch lesenswerten Aufsatz von Rudolf Hunziker: Der „Neue Berner-Kalender“ vor und unter der Redaktion Jeremias Gotthelfs. In: Neues Berner Taschenbuch auf das Jahr 1934. 39. Jg., 1933, S. 99-155, und Tschopp: Predigen, gefaBt in Lebenssprache (Anm. 1).

3 Vgl. Jeremias Gotthelf: Sämtliche Werke in 24 Bänden. Hrsg. von Rudolf Hunziker u. Hans Bloesch. Vierter Ergänzungsband. Bearb. von Kurt Guggisberg u. Werner Juker: Briefe. Erster Teil. ErlenbachZürich 1948 (im folgenden: EB 4), S. 282.

4 Vgl. Hunziker: Der "Neue Berner-Kalender“ (Anm. 2), S. $111 \mathrm{f}$.

5 Der Neue Bermer-Kalender für das Jahr 1840 lag bereits im Spätsommer 1839 vollständig vor (vgl. Hunziker: Der „Neue Berner-Kalender", Anm. 2, S. 112).

6 Die jeweiligen Publikationsorte der später an anderer Stelle veröffentlichten Beiträge zum Neuen Berner-Kalender werden im Anhang des 24. Bandes (S. 363-384) der Werkausgabe genannt (Jeremias 
seinem schriftstellerischen Werk abgekoppelte Nebenbeschäftigung; sie fügte sich vielmehr nahtlos in seine Selbstwahrnehmung als Autor ein, bot ihm jene Wirkungsmöglichkeiten, auf die er mit allen seinen Veröffentlichungen zielte. Sechs Jahre lang hat er den Neuen Berner-Kalender herausgegeben, hat alle darin erschienenen Beiträge verfaßt, sich mit dem Illustrator über die Bildausstattung geeinigt, den Buchdruckern eine bessere Papier- und Druckqualität abzuringen versucht. Als er sich schließlich, aus nicht eindeutig geklärten Gründen, dazu entschied, sein Amt als Kalendermacher niederzulegen, hat er dies, wie die hinterlassenen Entwürfe für den nicht mehr von ihm verantworteten Neuer Berner-Kalender auf das Jahr 1846 vermuten lassen, nicht leichten Herzens getan. ${ }^{7}$

In Kontrast zur hohen Signifikanz, die Gotthelf seinem Kalenderschaffen beimaß, steht, wie bereits angedeutet, die geringe Aufmerksamkeit, welche die Forschung dem Neuen Berner-Kalender zuteil werden ließ. Außer den immer noch grundlegenden Beiträgen Rudolf Hunzikers ${ }^{8}$ verdienen allenfalls einige in jüngster Zeit erschienene Aufsätze zu Kalendererzählungen Gotthelfs Erwähnung." Walter Muschgs Vorwort zu dem von ihm herausgegebenen Schatzkästlein des Neuen Berner-Kalenders ${ }^{10}$ und die einzige bisher veröffentlichte Dissertation zu Jeremias Gotthelfs Kalendertätigkeit $^{11}$ versprechen wenig wissenschaftlichen Gewinn, und dies gilt ebenso für die meist summarischen Bemerkungen in Darstellungen zum Werk des Emmentaler Dichters. ${ }^{12}$ Es sind nicht nur die auf den Neuen Berner-Kalender gerichteten Forschungsbemühungen, die sich als bisweilen ungenügend erweisen, kaum zu befriedigen vermag auch die Behandlung des Neuen Berner-Kalenders in der von Rudolf Hunziker und Hans Bloesch betreuten Werkausgabe, die durch willkürlich anmutende Weglassungen und Eingriffe in die sprachliche Gestalt des Textes gekennzeichnet ist. Problematisch ist außerdem die durch die Herausgeber vorgenommene neue Anordnung der Beiträge des erzählenden Teils, die den Textzusammenhang der einzelnen Jahrgänge auflöst und damit zugleich Spezifika des Mediums ,Kalender' unkenntlich werden läßt. In meinen nun folgenden Ausführungen soll es allerdings weder um eine systematische Auseinandersetzung mit der bisherigen Forschung zu Gotthelfs Kalenderschaffen noch um eine kritische Analyse der in der grundsätzlich verdienstvollen Gesamtausgabe der Werke Gotthelfs sich manifestierenden editorischen Praxis gehen.

Gotthelf: Sämtliche Werke in 24 Bänden. Hrsg. von Rudolf Hunziker u. Hans Bloesch. Bd. 23. Bearb. von Kurt Guggisberg und Werner Juker: Kalendergeschichten. Erster Teil. Erlenbach-Zürich 1931 [im folgenden SW XXIII] und Bd. 24. Bearb. von dens.: Kalendergeschichten. Zweiter Teil. ErlenbachZürich 1932 [im folgenden SW XXIV]).

Vgl. SW XXIV, S. $359 \mathrm{f}$.

* Vgl. Hunziker: Der "Neue Berner-Kalender" (Anm. 2). Von Rudolf Hunziker stammt auch der Kommentar zum Neuen Berner-Kalender in der von ihm mitherausgegebenen Edition sämtlicher Werke Gotthelfs.

9 Peter Utz: Gezügeltes Erzāhlen. Die beiden Fassungen von Gotthelfs Der Mordiofuhrmann. Ein Kalenderblat zu Gotthelfs zweihundertstem Geburtstag. In: DVjs 71, 1997, S. 589-606; sowie Tschopp: Predigen. gefaßt in Lebenssprache (Anm. 1).

10 Walter Muschg: Vorwort. In: Jeremias Gotthelf: Schatzkästlein des Neuen Berner Kalenders. Hrsg. von dems. Zürich 1943, S. 5-32.

If Hans Itten: Jeremias Gothelf als Kalenderschreiber. Diss. Bern 1957, Zürich 1959.

I2 Eine austūhrlichere Darstellung des Neuen Berner-Kalenders unter der Redaktion Gotthelfs findet sich in Ludwig Rohner: Kalendergeschichte und Kalender. Wiesbaden 1978, S. 327-339. 
Das Interesse gilt vielmehr Aspekten einer noch zu leistenden Kommentierung der von Gotthelf redigierten Kalenderjahrgänge. Dabei richte ich den Fokus auf drei Problemfelder, die sich mit der Kontextualisierung des Gotthelfschen Kalenderschaffens verbinden: In einem ersten Schritt gehe ich auf den Kalender als Mediengenre ein, um den Gattungskontext, in dem der Neue Berner-Kalender angesiedelt ist, zu erhellen. In einem zweiten Schritt stelle ich den Neuen Berner-Kalender in den Kontext der zeitgenössischen schweizerischen Kalenderproduktion. Besondere Relevanz kommt in diesem Zusammenhang der Frage zu, inwiefern sich Gotthelfs Neuer BernerKalender einem der in der ersten Hälfte des 19. Jahrhunderts dominierenden Kalendertypen zuordnen läßt bzw. was seine Singularität ausmacht. Abschließend werde ich in sehr knapper Form einige Überlegungen zum publizistischen Kontext des Neuen Berner-Kalenders formulieren. Konkret geht es um Interdependenzen, die sich aus Gotthelfs Funktion als Kalenderautor und seiner gleichzeitigen Tätigkeit als journalistischer Mitarbeiter des Berner Volksfreunds ergaben.

$\mathrm{Zu}$ den signifikanten Merkmalen des Kalenders gehört zum einen das, was Rudolf Schenda als ,Traditionsrelevanz $z^{6}$ bezeichnet hat, ${ }^{13}$ und zum andern dessen Ausrichtung auf einen vergleichsweise großen Adressatenkreis: ${ }^{14}$ Kalender zeichnen sich durch eine sowohl ihre formale als auch ihre inhaltliche Gestaltung determinierende Beharrungstendenz aus. Die seit dem 15. Jahrhundert gedruckten Einblattkalender finden ihre Fortsetzung im Kalendarium jener jährlich erscheinenden Drucke, die, um die so genannte ,Praktik' und einen erzählenden Teil bereichert, bereits im 16. bis 18 . und dann vor allem im 19. Jahrhundert eine zunehmend zahlreiche, meist ländliche Leserschaft erreichten. Die den praktischen Teil eines Kalenders konstituierenden Rubriken wie etwa Hundertjähriger Kalender oder Aderlaßtafel blieben über Jahrhunderte fest im Erwartungshorizont der Rezipienten verankert und überlebten zunächst auch die gegen den in den prognostischen und medizinischen Bestandteilen des Kalenders zutage tretenden Obskurantismus gerichteten Bemühungen der Volksaufklärer. Mit welchen Schwierigkeiten auch geringfügige konzeptionelle Modifikationen des Kalenders verbunden sein konnten, veranschaulicht ein 1829 in der Schweizerischen Monats-Chronik erschienener Artikel über „Die Schweizerischen Kalender". Der anonyme Verfasser, der seine volksaufklärerischen Ambitionen nicht verhehlt, moniert mit Blick auf den ansonsten wohlwollend beurteilten Basler Hinkenden Boten, daß das Aderlaßmännlein beibehalten wurde: „Dieses glaubte der Bote hersetzen zu müssen, weil,“ zitiert er den Basler Hinkenden Boten, ,"mancher Heyne und Joggle, Sepp und Rudi den Kopf schütteln würden, wenn das Aderlaßmännlein nicht im Kalender stünde, den sie kaufen wollen, sintemal es ihnen bis daher guten Rath gegeben haben soll, - zwar nur nach ihrer und des Schärers Meinung." "' Für die pragmatische Haltung des Verfassers des Basler Hinkenden Boten wird wenig Verständnis be-

13 Rudolf Schenda: Volk ohne Buch. Studien zur Sozialgeschichte der populären Lesestoffe 1770-1910. Frankfurt a. M. 1970 (Studien zur Philosophie und Literatur des neunzehnten Jahrhunderts. 5), S. $325 \mathrm{ff}$.

14 Aus der zunehmend reichhaltigeren Literatur zum Kalender sollen hier nur Rohner: Kalendergeschichte und Kalender (Anm. 12); Jan Knopf: Die deutsche Kalendergeschichte. Ein Arbeitsbuch. Frankfurt a. M. 1983; und Der Kalender als Fibel des Alltagswissens. Interkulturalität und populäre Aufklärung im 18. und 19. Jahrhundert. Hrsg. von York-Gothart Mix. Tübingen 2004 (Hallesche Beiträge zur Europäischen Aufklärung. 27) genannt werden. 
kundet. Dessen Vorgehen ließe sich, so der Rezensent, mit den Worten „Mahlet das Laster, - nur mahlet den Teufel dazu!" beschreiben und sei deshalb abzulehnen. ${ }^{15}$ Folgerichtig lobt er den offenkundig konsequenter einer volksaufklärerischen Programmatik verpflichteten Appenzeller Kalender als eine Publikation, die frei sei „von gemeiner Possenreißerey und abergläubischem Quarke ${ }^{\text {c. }}{ }^{16}$ Die Zitate belegen, daß die volkspädagogischen Visionen aufgeklärter Kalenderautoren und die Bedürfnisse der Kalenderleser nicht ohne weiteres in Einklang zu bringen waren. Der Wunsch der traditionsbewußten Rezipienten nach zwar veraltetem, in ihrer Wahrnehmung jedoch weiterhin autoritativem Wissen dürfte der Grund sein, weshalb auch in den von Gotthelf redigierten Jahrgängen des Neuen Berner-Kalenders die Aderlaßtafel beibehalten wurde und im Neuen Berner-Kalender auf das Jahr 1840 die in den späteren Jahrgängen getilgte Rubrik „Vom Schräpfen, Baden und Purgieren“ begegnet. Wer als Kalenderautor nicht riskieren wollte, daß seine Leser auf einem seit dem frühen 19. Jahrhundert zunehmend umkämpften publizistischen Markt zu Konkurrenzunternehmen wechselten, tat gut daran, die Erwartungen potentieller Käufer nicht völlig außer acht zu lassen und möglichst vieles von dem, was einen Kalender attraktiv machte, zu bieten.

Der Traditionalismus der Kalender hängt auch mit dem niedrigen Bildungsstand vieler Kalenderkäufer zusammen. Im ländlichen Raum war der Kalender noch bis weit ins 19. Jahrhundert hinein neben Bibel und Gesangbuch nicht selten die einzige Lektüre der bisweilen nur notdürftig alphabetisierten Bevölkerung. Er richtete sich an ein Publikum, dessen begrenzte literarische Erfahrung es geboten erscheinen ließ, Vertrautes nur sehr behutsam durch Neues zu ersetzen und in sprachlicher und thematischer Hinsicht nicht allzu voraussetzungsreich zu verfahren. Das Bemühen um eingängige Formulierungen, die sich an den Sprachgewohnheiten der Adressaten orientieren, und um eine Themenwahl, die deren Lesehorizont berücksichtigt, ist denn auch in den meisten Kalendern erkennbar.

Wer, wie dies beispielsweise Hans Itten getan hat, die Singularität des Neuem Berner-Kalenders postuliert, ${ }^{17}$ droht zu übersehen, in welchem Maße Gotthelf sich mit den durch die Gattungstradition vorgegebenen Vorstellungen und Erwartungen seiner Adressaten auseinandergesetzt hat. Auch ein nur oberflächlicher Blick in den ersten der von ihm betreuten Jahrgänge des Neuen Berner-Kalenders macht deutlich, daß die Leser das meiste von dem finden konnten, was ihnen auch andere zeitgenössische Kalender boten. Dies gilt naturgemäß vor allem für das Kalendarium und den praktischen Teil, der neben der schon erwähnten Aderlaßtafel und den Anweisungen zum Purgieren und Schröpfen die Rubrik „Von den Aspekten oder der Planetenstellung“, eine "Regenten-Liste", eine Tabelle, welche die Umrechnung von französischen in schweizerische Franken ermöglichte, sowie zwei Zinstabellen und natürlich die Jahrund die regelmäßigen Wochenmärkte enthält. Auch der erzählende Teil mit seinen nützlichen Informationen („Gemeinnütziges“" [S. 70-72; SW XXIII, S. 104-108];

15 Die Schweizerischen Kalender. In: Schweizerische Monaths-Chronik. Nr. 12. 14. Jg., 1829, S. 273-276. hier S. 274.

16 Ebd.. S. 276.

$17 \mathrm{Vgl}$. beispielsweise Itten: Gotthelf als Kalenderschreiber (Anm. 11). S. 9-21. 
„Die Erdflöhe“ [S. 72-73; SW XXIII, S. 108]) und seiner bunten Mischung aus rührenden, erbaulichen oder komischen Anekdoten und Erzählungen bewegt sich im Rahmen dessen, was die Rezipienten von einem populären Kalender erwarten durften. Die seit dem Neuen Berner-Kalender auf das Jahr 1841 regelmäßig abgedruckten ,Kuriositäten" wiederum knüpfen an an die bereits in frühneuzeitlichen Kalendern begegnenden ,Weltbegebenheiten ${ }^{6}{ }^{18}$ Nicht nur die konzeptionelle Ausrichtung des Neuen Berner-Kalenders, auch Gotthelfs brieflich überlieferte programmatische Überlegung offenbart sein Bemühen, die Erwartungen seiner Leser zu erfüllen. Wie wenig eine allein dem docere verpflichtete Vermittlung nützlichen Wissens dem Geschmack des Publikums entsprach, hat er scharfsichtig erkannt ${ }^{19}$ und gefordert, ein Kalenderautor müsse „das Leben, welches er beschreiben will, kennen aus eigener Anschauung ${ }^{\text {". }}{ }^{20}$ Darüber, daß es Gotthelf im Neuen Berner-Kalender nicht gelungen sei, Anspruch und Wirklichkeit zur Deckung zu bringen, bestand in der Forschung lange Konsens. Die bereits früh vertretene These, der eher mäßige Erfolg des Neuen Berner-Kalenders erkläre sich aus einem mangelnden Traditions- und Adressatenbezug, ist allerdings plausibel und problematisch zugleich. Wer wie Hunziker betont, Gotthelf habe als Verfasser und Herausgeber des Neuen Berner-Kalenders die Lesegewohnheiten seines Publikums nur ungenügend berücksichtigt, durch eine Fülle von Allusionen seine Leser nicht selten überfordert und sich durch die Schärfe, mit der er zu politischen Entwicklungen Stellung nahm, zahlreiche Feinde geschaffen, ${ }^{21}$ übersieht, daß in den 1840 er Jahren in der Schweiz gleich mehrere Kalender erschienen sind, die sich durch argumentative Komplexität und politische Polemik auszeichnen. Es genügt demnach nicht, den Neuen Berner-Kalender am tendenziell ahistorischen Modell eines idealtypischen Kalenders zu messen; aussagekräftiger als die Offenlegung von Gattungsbezügen scheint mir dessen Verortung innerhalb jener vielgestaltigen literarisch-publizistischen ,Landschaft' $\mathrm{zu}$ sein, die den in zeitlicher und regionaler Hinsicht unmittelbaren Kontext bildet.

Wenn im folgenden versucht wird, Gotthelfs Kalendertätigkeit historisch präziser zu lokalisieren, gilt es zunächst zwischen verschiedenen Formen von Kalendern zu differenzieren. Ungeachtet seiner grundsätzlichen Beharrungstendenz hat der Kalender in der ersten Hälfte des 19. Jahrhundert eine bemerkenswerte Diversifizierung erfahren. Zwar dominieren weiterhin die der frühneuzeitlichen Tradition verpflichteten und in aufklärerischem Sinne überformten Kalender, zugleich jedoch gewinnen die religiösen und die politischen Kalender an Bedeutung. Im Spannungsfeld der hier genannten, im interessierenden Zeitraum in der Schweiz verbreiteten Gattungstypen bewegt sich auch der Neue Berner-Kalender, der, wie gezeigt werden soll, sowohl

18 Vgl. Rohner: Kalendergeschichte und Kalender (Anm. 12), S. 330.

19 Vgl. Tschopp: Predigen, gefaßt in Lebenssprache (Anm. 1), S. 111-114.

20 Jeremias Gotthelf: Sämtliche Werke in 24 Bänden. Hrsg. von Rudolf Hunziker u. Hans Bloesch. Fünfter Ergänzungsband. Bearb. von Karl Guggisberg u. Wemer Juker: Briefe. Zweiter Teil. Erlenbach-Zürich 1949 (im folgenden: EB 5), S. 333. Zu Gotthelfs Konzeption des Volksschriftstellers vgl. Hanns Peter Holl: Gotthelf im Zeitgeflecht. Bauernleben, industrielle Revolution und Liberalismus in seinen Romanen. Tübingen 1985, S. 21-32.

21 Vgl. Hunziker: Der ,Neue Berner-Kalender“ (Anm. 2), S. 146-150. 
den volksaufklärerischen, als auch den religiösen und vor allem den politischen Kalendern in Analogie und Differenz verpflichtet ist.

Die Nähe des Neuen Berner-Kalenders zum Modell des volksaufklärerischen Kalenders ist offenkundig. ${ }^{22}$ Dies hängt nicht zuletzt mit der Genese des Neuen BernerKalenders zusammen, dessen erster Jahrgang im Herbst 1837 auf Veranlassung der Bernischen gemeinnützigen Gesellschaft publiziert worden war, die damit der in ihren Statuten als primärer Vereinszweck dekretierten „Beförderung der Volksbildung“ konkrete Gestalt zu verleihen beabsichtigte. ${ }^{23}$ Mit ihrem Anliegen befand sich die Bernische gemeinnützige Gesellschaft im übrigen auf der Höhe der Zeit, wie ein Blick in die bereits erwähnte, in der Schweizerischen Monats-Chronik erschienene Kalenderrezension zeigt. Dort nämlich hält der Verfasser gleich zu Beginn fest:

Die verständigsten Männer unserer Zeit sind darüber einig. daß die Kalender, als Volksbücher betrachtet, keineswegs eine unbedeutende Erscheinung, sondern der Beachtung und Vervollkommnung sehr werth sind. Gemeinnützige vaterländische Gesellschaften widmen diesem Gegenstande die verdiente Aufmerksamkeit, und gewiß nicht ohne heilsame Folgen. Der Kalender ist fast der einzige Weg, durch welchen nützliche Kenntnisse unter die niederste Volksklasse geleitet werden können: es wäre gewissenlos, wenn Volksfreunde diesen Weg nicht treulich benutzten. Es ist gewiß keine geringe Aufgabe, einen guten Kalender zu verfertigen, in welchem das Angenehme mit dem Nützlichen in gehörigem Verhältnisse steht. Belehrung soll allerdings Hauptzweck sein; dieser wird aber nicht erreicht, wenn nicht scherzhaft Unterhaltung mit kluger Wahl als Mittel gebraucht wird. Man gibt dem Kinde die Arzeney auf diejenige Art, auf welche es sie am liebsten nimmt. ${ }^{24}$

Auch die Bernische gemeinnützige Gesellschaft hat den von ihr initiierten Kalender von Beginn an als Vehikel einer dem Vernunfts- und dem Nützlichkeitspostulat verpflichteten Belehrung verstanden, wie der anonyme Herausgeber im Vorwort des Neuen Berner-Kalenders auf das Jahr 1838 deutlich macht. Sein "ganzes Bestreben“" sei dahin gegangen, ,nichts Unnützes in sein Buch aufzunehmen, nirgends durch Aufgeblasenheit zu verletzen, keinen seiner Leser durch Spott zu erbittern, durch an Prangerstellen zu erzürnen, oder durch Aufdeckung seiner Blößen dem Gelächter und der Schadenfreude Preis zu geben, ohne ihn dadurch zu bessern; daß ihm auch nicht zu thun war um Menschengunst, sondern darum, wie er durch verständige Belehrung, durch freundlichen wohlgemeinten Rath und schonende Liebe, einwirke auf Geist und Gemüth seiner Leser, und ihre Willenskraft bestimme, künftig nur das zu wählen, was des vernünftigen Menschen und Christen würdig ist." $\mathrm{Da \beta}$,verständige Belehrung" und "freundlicher wohlgemeinter Rath" allein noch keinen Absatz garantieren, zeigt die schwache Resonanz, die der Neue Berner-Kalender beim Publikum fand..$^{25}$ Zwar hat sich der Herausgeber durchaus bemüht, die landwirtschaftlichen und medizinischen Ratschläge, die naturkundlichen und historischen Abhandlungen und die mora-

22 Zur Bedeutung der (Volks)Aufk lärung für das Medium ,Kalender' vgl. Gehhardt Petrat: Einem besseren Dasein zu Diensten. Die Spur der Aufklärung im Medium Kalender zwischen 1700 und 1919. München u. a. 1991 (Deutsche Presseforschung. 27).

23 Vgl. Hunziker: Der „Neue Berner-Kalender“ (Anm. 2), S. 101.

24 Die schweizerischen Kalender (Anm. 15), S. 273.

25 Vgl. Hunziker: Der „Neue Berner-Kalender" (Anm. 2), S. 108. 
lisierenden Betrachtungen durch unterhaltende Einlagen aufzulockern. So finden sich neben Beiträgen wie „Medizinischer Nutzen des Honigs“, „Suwarow's Zug durch's Muotathal“ oder „Hauptgrundsätze eines verständigen Bauern“ auch einige Rätsel, Anekdoten, Aphorismen und Scharaden. Dennoch ist es dem Neuen Berner-Kalender auf das Jahr 1838 nicht gelungen, seine Leser zu gewinnen. Sein Verzicht auf Spekulatives und Sensationelles und seine von aufklärerischem Optimismus durchdrungene, gleichermaßen phantasie- und humorlose, sprachlich unbeholfen anmutende Diktion entsprachen offenkundig nicht den Erwartungen der Adressaten. Der geringe Erfolg des Unternehmens, der den Vorstand der Bernischen gemeinnützigen Gesellschaft dazu bewog, Gotthelf die Kalenderredaktion anzutragen, hatte zunächst allerdings keinen Einfluß auf die volkspädagogische Ausrichtung des Neuen Berner-Kalenders, nicht zuletzt deshalb, weil Gotthelfs Auffassung eines gelungenen Kalenders den Intentionen seiner Auftraggeber nicht grundsätzlich widersprach. Wenn er sich mit Blick auf die von ihm verantworteten Jahrgänge des Neuen Berner-Kalenders von "gemeinnützigen Langeweilbehältern" distanziert, ${ }^{26}$ bedeutet dies keine prinzipielle Absage an das Modell des volksaufklärerischen Kalenders. Die polemische Spitze richtet sich vielmehr gegen jene gut gemeinten Elaborate, zu denen auch die ersten beiden Jahrgänge des Neuen Berner-Kalenders zu zählen sind, die ihrem Publikum weder substantielle Belehrung noch Unterhaltung zu bieten vermochten und deshalb die Lektüregewohnheiten und -erwartungen der Rezipienten verfehlten. Mit seinem Neuen Berner-Kalender zielt Gotthelf demnach nicht auf die Überwindung des Gattungstypus ,volksaufklärerischer Kalender', sondem auf dessen Reform im Sinne einer Adaption an zeitgenössische Lesebedürfnisse. Daß Gotthelfs Neuer BernerKalender bei aller Eigenwilligkeit, die ihm in der germanistischen Forschung zu Recht attestiert wurde, ${ }^{27}$ die Anforderungen an einen auf Aufklänung bedachten populären Kalender im Blick behielt, zeigt der Vergleich mit dessen schärfstem Konkurrenten, dem von Gottlieb Jakob Kuhn herausgegebenen Berner Hinkenden Boten.

Mit dem Pfarrer und Liederdichter Kuhn verbindet Gotthelf die Skepsis einem zu einseitig auf Didaxe bedachten Kalender gegenüber. Wie Gotthelf hat auch Kuhn nicht mit Kritik am Ergebnis der publizistischen Bemühungen der „Alleinweisen“, ${ }^{28}$ wie er die Vorstandsmitglieder der Bernischen gemeinnützigen Gesellschaft ironisch bezeichnete, gespart. Der Kalender sei nicht nach dem "Geschmacke [des] Volkes“, habe „zu viel Schulmeisterliches, Trockenes, zu wenig Heiterkeit und Salz“. ${ }^{29}$ Zwar spricht sich der erfahrene Kalendermacher Kuhn nicht grundsätzlich gegen das „Kluge" und das „Nützliche“ aus, entscheidend sei jedoch, daß ein an breitere Publikums-

26 Vgl. den Brief vom 16.12.1838 an Carl Bitzius, in dem er betont: „Es gibt einen eigenen Kalender. keinen zusammengetragenen aus Naturgeschichten und andern gemeinnützigen Langeweilbehältem. Aus Rezepten, wie Wanzen zu vertreiben seien, und wie viel Junge die Steinböckin habe, macht man keinen vemünftigen Kalender. Das kömmt aus der verflucht dummen gemeinnützigen Zeit. wo man im Ernste des Lebens nicht tiefer kam als zu Rezepten und in der jetzt noch unsere Staatsmänner taumeln. Ich mōchte in den Kalender Predigen bringen, d.h. hohe Wahrheiten, aber entkleidet von allem Kirchlichen. gefaßt in Lebenssprache, wie man sie auf der Kanzel nicht duldet." (EB 4, S. 28If.).

27 Vgl. etwa Hunziker: Der „Neue Berner-Kalender” (Anm. 2), S. 146-150.

28 Zitiert nach ebd., S. 102.

29 Ebd., S. 107. 
schichten gerichtetes Periodikum den „Volkston nicht verliere“, ${ }^{30}$ denn, „[w]enn man will, daß der Kalender unter dem Volke kursiere und Interesse finde, so muß man mit dem Volke seine Sprache reden“.31 Dafür, daß ihm dies mit seinem eigenen Kalender gelungen ist, spricht die weite Verbreitung des Berner Hinkenden Boten, der in der Forschung zu Recht als ebenso repräsentativer wie populärer Kalender beurteilt wurde. ${ }^{32}$ Der Historische Kalender oder Hinkende Bot auf das Jahr 1839,,33 der unmittelbar bevor Gotthelf seine Kalendertätigkeit aufnahm, erschienen ist, verdeutlicht beispielhaft Kuhns Bemühen um einen Kalender, der die Bedürfnisse seines Publikums erfüllt, ohne auf Aufklärung zu verzichten. Der kalendarische Teil bietet dem Leser neben dem Alten und Neuen Kalender und den einzelnen Tagen zugeordneten Planetenstellungen eine Aufzählung der Jahrmärkte, eine Regentenliste (,Die gekrönten

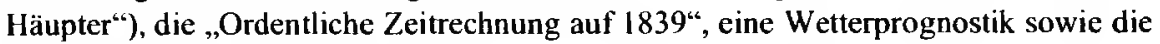
traditionellen Rubriken „Von den Finsternissen“, „Von der Fruchtbarkeit“, "Von den Krankheiten“ und „Von dem Krieg“. In den praktischen Teil integriert sind außerdem mit humoristischen Vierzeilern versehene Monatsvignetten, „Haus- und Bauernregeln“, Rätsel, Sinnsprüche, Gedichte sowie eine Fabel. Der Erzählteil ist ebenso umfangreich wie formal und inhaltlich vielgestaltig. Naturkundliches (z. B. „Die Sonne“, „Die Gemse“ oder „Die Baumwolle“), Historisches (z. B. „Die Schlacht bei Laupen 1339") oder Enzyklopädisches („Wörterbuch") findet der Leser ebenso wie Rezepte und Ratschläge (z. B. „Etwas vom Bücherkaufen und Lesen" oder „Die Hundetaxe"), Gespenstergeschichten („Lustig bezahlt", „Schmerzlich bezahlt", „Noch schlimmer") und zahlreiche Anekdoten. Bemerkenswert - und darin unterscheidet sich Kuhn fundamental von Gotthelf - ist der betont unpolemische Charakter der Darstellung. Die Gesinnung des konservativen Kuhn wird zwar etwa dort erkennbar, wo er einen radikalen Parteigänger schildert, der zu Hause seine Frau aufs Ärgste tyrannisiert („Ein Nachtstück"), zugleich jedoch bemüht sich Kuhn in politischen und konfessionellen Fragen um eine differenzierte und tendenziell gemäßigte Haltung. Exemplarisch zeigt dies die Auseinandersetzung mit kirchlich nicht gebundenen religiösen Gemeinschaften im Beitrag „Etwas vom Sektenwesen“, den er mit folgenden Worten einleitet:

Man hat den Boten schon hie und da angestochen, er solle doch einmal die Stündeler, Sektirer, Neutäufer und dergleichen Leute in seinem Kalender recht durchhecheln. Aber das will er nicht thun, und zwar darum nicht: 1) Es betrifft diese Sache die Religion, und die ist zu ehrwürdig und $z u$ heilig, als daß sie im Kalender besprochen werden sollte. 2) Mit Spotten und Lächerlichmachen werden die Leute nur erzürnt, aber weder belehrt noch bekehrt. 3)

30 Ebd., S. 102.

31 Ebd., S. 104.

32 Vgl. ebd.. S. 107f. Kuhn hat den Kalender von 1804-1810 und von 1833-1844 redaktionell betreut (Hunziker: Der „Neue Berner-Kalender“, Anm. 2, S. 101, Anm. 10). Zur Biographie Kuhns vgl. Heinrich Stickelberger: Der Volksdichter Gottlieb Jakob Kuhn 1775-1849. Bern 1909 (Neujabrs-Blatt der literarischen Gesellschaft Bern auf das Jahr 1910); zum Berner Hinkenden Boten vgl. Jules Capré: Histoire du véritable Messager boiteux de Berne et Vevey. 2 Bde. Vevey 1884/1888; sowie [Johann Heinrich Graf]: Historischer Kalender oder der Hinkende Bot. Seine Entstehung und Geschichte. Ein Beitrag zur bernischen Buchdrucker- und Kalendergeschichte. Hrsg. von der Stämpflischen Buchdruckerei. Bern 1896.

33 Der vollständige Titel lautet: Historischer Kalender oder Hinkender Bot, auf das Jahr 1839. Bern, bei Carl Stämpfli, Buchdrucker. 
Zum Belehren und Bekehren ist aber der Bote nicht der rechte Mann, und ist immer der Meinung, es stünde viel besser in der Welt, wenn jeder steif bei seiner Sache blicbe, und seine Nase nicht in anderer Leute Beruf und Amt steckte. ${ }^{34}$

Es ist die hier zum Ausdruck gelangende auf Ausgleich bedachte Haltung, es sind die formale und inhaltliche Vielfalt der Beiträge, eine souveräne Sprache, die in der direkten Rede oft in Dialekt übergeht, sowie der Witz, in den die Belehrungen gekleidet sind, die dem Berner Hinkenden Boten das Interesse der Leser gesichert haben. An die meisterhafte Erzählkunst eines Johann Peter Hebel reichen Kuhns Kalenderstücke nicht heran, in seiner Gesamtheit bildet der Berner Hinkende Bote nichtsdestotrotz das Musterbeispiel eines Kalenders, der Belehrung und Unterhaltung im Gleichgewicht zu halten und im Dialog mit den Lesern den richtigen Ton zu treffen vermag.

Der erste von Gotthelf alleinverantwortlich redigierte Jahrgang des Neuen BernerKalenders unterscheidet sich auf den ersten Blick nur wenig von anderen zeitgleich publizierten Kalendern. ${ }^{35}$ Der Neue Berner-Kalender auf das Jahr 1840 entspricht sowohl hinsichtlich seines Titelblatts als auch hinsichtlich seiner Aufmachung den Ausgaben von 1838 und 1839; das Kalendarium wurde in nur geringfügig modifizierter Form übernommen. Auch der Erzählteil enthält zunächst wenig Überraschendes: Naturkundliches verspricht das „Das Krokodill" (NBK [1840], S. 50-54; SW XXIII, S. 58-67) oder „Das Wetter" (NBK [1840], S. 73-74; SW XXIII, S. 95-98), Nütz]iches die unter dem Titel „Gemeinnütziges“ (NBK [1840], S. 70-72; SW XXIII, S. 104-108) gesammelten Rezepte; Berichterstattung über aktuelle Begebenheiten (z. B. „Einiges aus dem eidgenössischen Lustlager zu Sursee“ [NBK [1840], S. 3133; SW XXIII, S. 22-28]), Anekdoten (2. B. „Mißverständnisse“ [NBK [1840], S. 43; SW XXIII, S. 101f.] oder „Fatale Zumuthung“ [NBK [1840], S. 43; SW XXIII, S. 100]) oder moralische Exempla (z. B. "Bedenkliche Verlegenheit" [NBK [1840], S. 49-50; SW XXIII, S. 54-58]) begegnen auch im Berner Hinkenden Boten. Bei näherer Betrachtung wird allerdings deutlich, daß die hier beispielhaft genannten Kalenderbeiträge im Titel Erwartungen evozieren, die der Text nicht einlöst. So handelt es sich bei „Das Krokodill“ nicht um eine zoologische Abhandlung, sondern um die satirische Entlarvung menschlicher Leichtgläubigkeit; „Das Wetter" wiederum bietet weniger eine meteorologische als vielmehr eine theologische Betrachtung, und „Einiges aus dem eidgenössischen Lustlager zu Sursee“ spart bei allem patriotischen Ernst nicht mit kritisch-ironischen Seitenhieben. Ich habe an anderer Stelle dargelegt, $\mathrm{da} ß$ es die Verbindung von erbaulicher und satirischer Schreibart, von religiöser Ermahnung und Sozialkritik ist, welche die Eigenart von Gotthelfs Kalender ausmacht. ${ }^{36}$ In seiner sich bereits im ersten von ihm redigierten Jahrgang des Neuen BernerKalenders manifestierenden Vorliebe für die Paränese ${ }^{37}$ und seinem keinesfalls aus-

34 Ebd., fol. $\mathrm{G}^{\mathrm{r}}$

35 Vgl. Tschopp: Predigen, gefaßt in Lebenssprache (Anm. 1), S. $114 f$.

36 Vgl. ebd., S. II5-118.

37 Auf geistliche Ermahnung zielen die Homilie „Der Glaube“ (NBK [1840], S. 27-31; SW XXIII. S. 1222), die den Auftakt für einen Predigtryklus bildet, der in den folgenden Kalenderjahrgängen mit ..Die Hoffnung" (NBK [1841], S. 27-31; SW XXIII, S. 119-128), ,Die Liebe“" (NBK [1842]. S. 42-47: SW XXIII, S. 260-272), „Die Furcht* (NBK [1843], S. 27-31; SW XXIII, S. 334-345), ..Demut" (NBK [1844], S. 51-58; SW XXIV. S. 61-75) und "Die Sanftmut" (NBK [1845]. S.41-49: SW XXIV. 
schließlich auf die ab 1841 den Erzählteil eröffnenden ,Kuriositäten' beschränkten Hang zu satirischer Entlarvung und polemischer Attacke unterscheidet sich Gotthelf in der Tat von Kalenderautoren wie Kuhn. In dem Maße allerdings, in dem sich der Emmentaler Pfarrer im Neuen Berner-Kalender vom Ideal eines aufklärerischen Kalenders entfernt, nähert er sich den religiösen und politischen Kalendern an, die in der Schweiz in der ersten Hälfte des 19. Jahrhunderts ebenfalls ihr Publikum fanden.

Ohne dies näher zu begründen, hat Ursula Brunold-Bigler den Neuen BernerKalender unter die religiösen Kalender subsumiert. ${ }^{38} \mathrm{Da}$ sie in ihrer Studie auf eine Definition des Terminus, religiöser Kalender' verzichtet, bleibt offen, welche Überlegungen der von ihr gewählten typologischen Zuordnung zugrunde lagen. Wenn Gotthelf zu Beginn seiner redaktionellen Tätigkeit in einem Brief an Carl Bitzius die programmatische Absicht bekundet, in seinem Kalender „Predigen bringen [zu wollen], d. h. hohe Wahrheiten, aber entkleidet von allem Kirchlichen, gefaßt in Lebenssprache, wie man sie auf der Kanzel nicht duldet, "39 und den Erzählteil des ersten von ihm betreuten Jahrgangs mit einer Homilie eröffnet, mag in der Tat der Eindruck entstehen, der Neue Berner-Kalender verfolge in erster Linie eine religiöse Intention. Ihn deshalb den meist von kirchlichen Trägern herausgegebenen konfessionell gebundenen Kalendern zuzuordnen, scheint mir dennoch problematisch, unterscheidet er sich von diesen doch in entscheidender Hinsicht, wie der Vergleich mit dem ebenfalls in Bern erscheinenden Christlichen Hausfreund deutlich macht. ${ }^{40}$ Der von der 1831 gegründeten, pietistisch geprägten Evangelischen Gesellschaft initiierte Christliche Hausfreund, der es auf insgesamt acht Jahrgänge brachte (1835-1843), verstand sich als Alternative zu jenen Kalendern, die, wie es in einem Jahresbericht der Evangelischen Gesellschaft heißt, „dem Weltgeist fröhnen, und durch ihre oft ärgerlichen und unsittlichen Geschichten mehr schaden als nützen“. ${ }^{41}$ Als „Christ-Bote“42 sah er es einerseits als seine Aufgabe an, gegen die säkularen Auswüchse der Aufklärung und das im Kanton Bern verbreitete Sektenwesen zu kämpfen, andererseits und vor allem wollte er seine Leser in ihrem Glauben bestärken und sie zu gottgefälligem Denken und Handeln anweisen. Der Christliche Hausfreund auf das Jahr $1839^{43}$ offenbart seine religiöse Zielsetzung bereits im Titelblatt, das weder eine Vedute, noch Szenen aus der schweizerischen Geschichte oder die Darstellung des Hinkenden Boten zeigt, sondern einen über den Acker gehenden Sämann abbildet. Das unter dem Holzschnitt

S. 152-169) eine Fortsetzung findet. Erbaulichen Charakter haben außerdem „Seltsamer Trost“ (NBK [1840], Kalendarium; SW XXIII, S. 6-12) und ,Das Wetter" (NBK [1840], S. 73-74; SW XXIII, S. 95-98) sowie die legendenhaften Erzählungen „Der bekehrte Mordiofuhmmann“ (NBK [1840], S. 35 4I; SW XXIII, S. 31 42), „Der russische Knabe “ (NBK [1840], S. 47 48; SW XXIII, S. 5I-54), „Das gelbe Vōgelein und das arme Margrithli“ (NBK [1840], S. 55-60; SW XXIIl, S. 67-77) und „Die beiden Raben und der Holzschelm“" (NBK [1840], S. 62-66; SW XXIII, S. 79-88).

38 Ursula Brunold-Bigler: Die religiösen Volkskalender der Schweiz im 19. Jahrhundert. Basel 1982 (Beiträge zur Volkskunde. 2), S. 55f. Im folgenden bezeichnet der Begriff in der Regel durch eine (frei)kirchliche Trägerschaft verantwortete, konfessionell gebundene Kalender mit primär erbaulichem Charakter.

Vgl. Anm. 26.

40 Zum Christlichen Hausfreund vgl. Brunold-Bigler: Die religiösen Volkskalender (Anm. 38), S. 49-52.

41 Zitiert nach ebd., S. 50.

4? Ebd.

43 Der vollständige Titel lautet: Der christliche Hausfreund. Für das Jahr 1839. Bei Carl Wüterich, Buchbinder, Nachfolger von Fr. Gaudard. 
befindliche Zitat (,Etliches fiel auf ein gut Land. Matth. 13,8.“) verweist auf jene biblische Parabel, welche die Vorlage für die Illustration bildet. Im Innern unterscheidet sich der Christliche Hausfreund auf den ersten Blick nicht stark von zeitgenössischen volksaufklärerischen Kalendern. Der praktische Teil enthält neben dem Kalendarium mit dem Alten und Neuen Kalender sowie den Planetenstellungen die Monatsvignetten, die Jahr- und regelmäßigen Wochenmärkte, die auch in andern Kalendern abgedruckte „Regenten-Liste“, eine „Fest- und astronomische Zeitrechnung für das Jahr 1839“, eine Währungs- und zwei Zinstabellen. Immerhin indizieren der für jeden Tag vorgesehene Bibelvers, die in die Praktik integrierten Kurzbiographien bedeutender Reformatoren und die theologische Behandlung der Rubriken „Von der Fruchtbarkeit der Erde“, „Von Krankheiten“, „Vom Krieg“, „Von der Witterung" die religiöse Ausrichtung der Publikation. Fast durchwegs erbaulicher Natur sind auch die den Erzählteil konstituierenden Kalenderbeiträge: Lebenspraktischen Nutzen haben nur gerade „Die heilsamen Wirkungen des kalten Wassers" und „Ueber das Aufblähen der wiederkauenden Hausthiere", ansonsten enthält der Christliche Hausfreund auf das Jahr 1839 neben einem Missionsbericht (,Züge aus dem Leben des Missionars Christian Friedrich Schwartz") und einem Beitrag zur Kirchenhistorie („Kurze Geschichte der Kirche Christi in den ersten drei Jahrhunderten“) vor allem geistliche Ermahnungen (z. B. „Heiligung des Sabbaths", „Ein Wort über die Unkeuschheit“ oder „Der Geiz ist eine Wurzel alles Uebels. 1. Tim. 6,10“). Auf Abschreckung zielen die Darstellung der gottlosen Stadt Ninive und die drei abschlieBenden Beiträge, die veranschaulichen, wie Gott die Spötter bestraft. Kennzeichnend für den Christlichen Hausfreund ist demnach dessen konsequent erbaulicher Charakter. Unterhaltende Elemente fehlen gänzlich, die Ratschläge für Landwirte und die medizinischen Hinweise sind auf ein Minimum reduziert; der Schwerpunkt liegt auf der Vermittlung eines christlich fundierten Normensystems und, wie die in den Kalenderjahrgängen rekurrierenden Berichte über Missionare, kirchengeschichtlichen Abhandlungen und Lebensbilder protestantischer Theologen belegen, der Stärkung evangelischer Identität.

Aus der knappen Beschreibung eines - repräsentativen - Jahrgangs des Christlichen Hausfreunds dürfte ersichtlich geworden sein, daß Gotthelfs Neuer BernerKalender sich in mehrfacher Hinsicht von diesem unterscheidet. Ungeachtet der Homilien „Der Glaube“ (1840), „Die Hoffnung“ (1841), „Die Liebe“ (1842), „Die Furcht" (1843), „Demut“ (1844) und „Die Sanftmut“ (1845), ungeachtet auch einer bisweilen offenkundigen Vorliebe für die erbauliche Schreibart, läßt sich der Neuen Berner-Kalender demnach nicht ohne weiteres den religiösen Kalendern zuordnen. Gegen eine derartige Zuordnung sprechen zum einen der nicht nur für die primär unterhaltenden Anekdoten und Erzählungen charakteristische ironische Ton und zum andern die dezidierten politischen Stellungnahmen, die im Christlichen Hausfreund geradezu ängstlich gemieden werden.

Letztere nun lassen es sinnvoll erscheinen, einen dritten Kalendertypus in den Blick zu nehmen, dessen Einfluß auf Gotthelfs Neuen Berner-Kalender so offenkundig wie unerforscht ist: den politischen Kalender. ,Politischer Kalender meint in diesem Zusammenhang eine im 19. Jahrhundert an Bedeutung gewinnende Sonder- 
form des Kalenders, deren Entstehung sich der Dynamisierung des politischen Diskurses im Zuge verstärkter republikanischer Bemühungen verdankt. Kennzeichnend für die in der ersten Hälfte des 19. Jahrhunderts veröffentlichten politischen Kalender ist deren zeitliche und räumliche Fokussierung; sie waren das meist kurzlebige Ergebnis einer spezifischen, zeitlich begrenzten politischen Konstellation und beschränkten sich in der Regel auf jenen Raum, in dem diese Konstellation die offentliche Kommunikation determinierte. Es ist so gesehen kein Zufall, daß sich die Schweiz in den 1840 er Jahren als besonders fruchtbarer Boden für politisch intendierte Kalenderprojekte erwies. Der politische Antagonismus zwischen Liberalen, Radikalen und Konservativen generierte öffentlich ausgetragene Debatten, die den publizistischen Markt in hohem Maße beflügelten. Da die Reichweite der ohnehin seltenen Zensurmaßnahmen auch in den konservativ regierten Kantonen begrenzt war, kam es nicht nur zur Neugründung zahlreicher Periodika, sondern auch zu einer Politisierung bereits bestehender Medien. Die Verschärfung der Auseinandersetzungen zwischen den freisinnigen und den konservativen Parteigängern seit Ende der 1830er Jahre, die in den Freischarenzügen und schließlich im Sonderbundskrieg, aus dem 1848 der Schweizerische Bundesstaat hervorging, kulminierten, spiegelt sich auch und gerade in zeitgenössischen Kalendern wie etwa dem Gukkasten-Kalender ${ }^{44}$ und vor allem dem Schweizerischen Bilderkalender, der im folgenden einer näheren Betrachtung unterzogen werden soll.

Als "Pendant" zum seit 1839 erscheinenden Schweizerischen Bilderkalender hat Winfried Bauer Gotthelfs Neuen Berner-Kalender bezeichnet. ${ }^{45}$ In der Tat ist es plausibel, Gotthelfs Kalender als Reaktion auf den nach seinem Herausgeber auch ,Disteli-Kalender' genannten Schweizerischen Bilderkalender zu deuten. Der konzeptionelle Wandel, dem der Neue Berner-Kalender zwischen 1840 und 1845 unterliegt, dürfte sich nicht zuletzt aus Gotthelfs Auseinandersetzung mit dem ebenso beliebten wie umstrittenen Kalender seines politischen Gegners erklären. ${ }^{46}$ Als Gotthelf entschied, die ihm angetragene Kalenderredaktion anzunehmen, lag der vom Solothumer Maler und Zeichner Martin Disteli verantwortete erste Jahrgang des Schweizerischen Bilderkalenders bereits vor. ${ }^{47}$ Seine Besonderheit zeigt sich weniger im praktischen Teil, der neben dem Katholischen und Reformierten Kalender eine „Fest- und astronomische

44 Der entschieden radikale Gukkasten-Kalender ist 1844 erstmals unter dem Titel Gukkasten-Kalender für das Jahr, nicht der Gnade sondern hoffentlich des Rechts und der Vernumft, 1845 erschienen und erlebte insgesamt nur zwei Jahrgänge. Verfasser war der Sohn von Gotthelfs Verleger Christian Albrech Jenni, Friedrich Jenni, der auch und vor allem als Herausgeber der zwischen 1840 und 1849 veröffentlichten satirischen Wochenzeitung Gukkasten Berühmtheit erlangt hatte.

45

Winfried Bauer: Jeremias Gotthelf. Ein Vertreter der geistlichen Restauration der Biedermeierzeit. Stuttgart u. a. 1975 (Studien zur Poetik und Geschichte der Literatur. 4I), S. 148.

46 Dass Gottheif den Schweizerischen Bilderkalender kannte, geht aus einem Brief vom 16.11.1844 an Bernhard Rudolf Fetscherin hervor, in dem er ausdrücklich auf Distelis Vetter Hammer Bezug nimm (Jeremias Gotthelf: Sämtliche Werke in 24 Bänden. Hrsg. von Rudolf Hunziker u. Hans Bloesch. Sechster Ergānzungsband. Bearb. von Kurt Guggisberg und Wemer Juker: Briefe. Dritter Teil. ErlenbachZürich 1950, S. 129).

47 Der vollstândige Titel lautet: Schweizerischer Bilderkalender für das Jahr 1839 von M. Disteli. Solothurn, Druck und Verlag Gassmann, Sohn. Zum ,Disteli-Kalender 'vgl. Adolf Lechner: Der Disteli- und der Ziegler-Kalender. Eine bibliographische und historische Studie. Solothum 1911; und Gottfried Wälchli: Martin Disteli 1802-1844. Zeit - Leben-Werk. Zürich 1943, S. 53-65. 
Zeitrechnung für das Jahr $1839 “$, ein Verzeichnis der Jahr- und regelmäßigen Wochenmärkte, eine „Regenten-Liste“ sowie die Rubriken „Von den Jahreszeiten“ und "Von den dießjährigen Finsternissen" enthält. Ungewöhnlich ist immerhin die in die Praktik integrierte „Zeitrechnung der schweizerischen Freiheit" sowie eine statistische Angaben zur politischen und geographischen Gliederung, zur Bevölkerung sowie zu den konfessionellen Verhältnissen enthaltende „Uebersichtstafel der XXII Kantone der Eidgenossenschaft". Sie stehen in Einklang mit jener politisch-patriotischen Tendenz, die auch das Titelblatt mit seiner Abbildung des Drachentöters Struthan Winkelried und der Darstellung der Erschießung Gesslers durch Wilhelm Tell verrät. Es sind jedoch vor allem der Erzählteil und die mit ihm verbundenen Illustrationen, die die politische Zielsetzung des Schweizerischen Bilderkalenders erhellen. Den Erzählteil eröffnen „Bilder aus dem Bauernkrieg von 1653“, die aus radikaler Sicht die bäuerliche Revolte als berechtigten, wenn auch schließlich gescheiterten Kampf gegen die Unterdrückung durch eine auf ihre Privilegien bedachte Obrigkeit beschreiben. Es folgen die Darstellung eines politischen Konflikts aus der jüngsten schweizerischen Vergangenheit (,Der Hörner und Klauen Streit auf der Landsgemeinde am Rothenthurm, den 6. Mai 1838“), die satirische Abrechnung mit den in der historischen Figur des Hauptmann Hammer personifizierten Angehörigen der konservativen Partei (,Kurze und faßliche Beschreibung der Lebensgeschichte meines Herrn Vetters"), drei anekdotische Beiträge („Der Geizhals“, „Der Schuldirektor und der Schulmeister" und „O du Bart! O du Zopf!") sowie schließlich eine entomologische Abhandlung (,Einiges über die Verwandlung der Insekten“).

Die bemerkenswerte Resonanz, auf die der Schweizerische Bilderkalender in der gesamten deutschsprachigen Schweiz stieß - die Auflage erreichte bereits $184020^{\prime} 000$ Exemplare $^{48}$ - ist insofern überraschend, als er nur sehr wenig von dem enthält, was einer aufklärerischen Programmatik verpflichtete populäre Kalender auszeichnete. Anders als die Mehrzahl seiner zeitgenössischen Konkurrenten bot der Schweizerische Bilderkalender kaum Nützliches und nur vereinzelt Anekdotisches; die Textbeiträge konfrontierten die Leser vielmehr mit vaterländischer Geschichte, mil Ereignissen der unmittelbaren Gegenwart sowie mit politischer Satire. Man hat den Erfolg des Schweizerischen Bilderkalenders vor allem mit den darin abgedruckten Illustrationen begründet, deren ungewöhnliche Qualität sogar von Distelis konservativen Kritikern anerkannt wurde. ${ }^{49}$ Die Bildausstattung ist in der Tat nicht nur in quantitativer Hinsicht außergewöhnlich: Neben den Monatsvignetten im Kalendarium (seit 1842) weist der Schweizerische Bilderkalender großformatige Lithographien auf, welche Distelis künstlerische Meisterschaft auf beeindruckende Weise unter Beweis stellen. In nicht geringerem Maße als der offenkundige ästhetische Mehrwert, den der Schweizerische Bilderkalender seinem Publikum versprach, dürfte allerdings dessen politische Tendenz den Absatz gefördert haben. Sowohl die von Peter Felber, Redakteur des Solo-

48 Zur Entwicklung der Auflage des ,Disteli-Kalenders' vgl. Lucien Leitess. Irma Noseda u. Bemhard Wiebel: Martin Disteli 1802-1844. ...und fluchend steht das Volk vor seinen Bildem. Olten 1981. S. 85.

49 So hält der Verfasser eines in der Nummer vom 1. Februar 1844 erschienenen Arikels in der Luzener Staatszeitung, die sich mit Angriffen gegen den Schweizerischen Bilderkalender besonders hervorat. mit Blick auf den Zeichner Disteli fest. es sei „ewig schade, da $B$ ein unleugbares Talent in diese Pfützen sich verloren“" (zitiert nach: Wälchli: Martin Disteli 1802-1844, Anm. 47. S. 64). 
thurner Blattes und Regierungsrat, verfaßten Textbeiträge als auch die in den späteren Jahrgängen zunehmend zur Karikatur neigenden Illustrationen stellten sich ganz in den Dienst des politischen Radikalismus. Noch stärker als im ersten dienten die in den zwischen 1840 und 1844 veröffentlichten Kalenderjahrgängen abgedruckten historischen Darstellungen und politischen Invektiven dazu, in einem Moment erregter politischer Debatten die Stimme zugunsten einer demokratischen, politisch fortschrittlichen Schweiz zu erheben. Die sich in Bild und Text manifestierende polemische Schärfe, mit der dies geschah, hat denn auch zu heftigen Reaktionen in den konservativen Kantonen geführt. Gegen den Radikalismus und Antiklerikalismus des Schweizerischen Bilderkalenders verwehrten sich nicht nur konservative Presseorgane, auch einige kantonale Behörden wurden aktiv und versuchten, mit allerdings geringem Erfolg, Distelis populäre Publikation zu verbieten. ${ }^{50}$ Die massiven Interventionen gegen den Schweizerischen Bilderkalender sind, ebenso wie die dezidierte Unterstützung, die er in der freisinnigen Presse erfuhr, ${ }^{51}$ ein Indiz für die Polarisierung der Schweiz im Vorfeld der Staatsgründung, eine Polarisierung, die, wie auch Gotthelfs Neuer Berner-Kalender belegt, für die Druckmedien nicht ohne Folgen blieb.

Die politisch motivierte Polemik in Gotthelfs Neuem Berner-Kalender ist, dies macht der Blick auf den Schweizerischen Bilderkalender deutlich, keinesfalls derart singulär, wie dies in der Forschung bisweilen behauptet wurde. Die kritische Auseinandersetzung mit den Reformbemühungen der radikalen Berner Regierung, zu nennen wäre hier etwa die Erzählung Die Schelmenzucht aus dem Neuen Berner-Kalender für das Jahr 1840, ${ }^{52}$ vor allem jedoch die scharfzüngige Abrechnung mit freisinnigen Tendenzen in den seit 1842 den Erzählteil eröffnenden „Kuriositäten“ bilden den Versuch, der durch radikale Parteigänger im Medium des Kalenders bewerkstelligten Agitation eine andere, konservativere Sichtweise entgegenzusetzen. Wenn Gotthelf dabei nicht mit Polemik spart und seinem satirischen Talent keine Zügel anlegt, was ihm wohlgesinnte und eng verbundene Freunde wie Carl Bitzius wiederholt zu mahnenden Briefen veranlaßte, ${ }^{53}$ steht dies in Einklang mit dem aufgeheizten Diskursklima der frühen 1840er Jahre. In welch scharfem Ton politische Gegner angegriffen werden konnten, belegen nicht zuletzt jene Zeitungsartikel, in denen Gotthelfs Neuer Berner-Kalender einer in doppeltem Sinn radikalen Kritik unterzogen wurde. So spart ein im Schweizerischen Beobachter vom 8. Oktober 1844 erschienener Artikel nicht mit Anwürfen gegen den eben in den Handel gelangten Neuen Berner-Kalender für das Jahr 1845: Nachdem der mit „Ein Altberner“ firmierende Verfasser ironisch vorgeschlagen hat, eine vakant gewordene Professur an der Berner Hochschule mit Gotthelf zu besetzen, fährt er fort:

$50 \mathrm{Zu}$ den in den Kantonen Obwalden und Luzern gegen Martin Disteli geführten Prozessen vgl. Lechner: Der Disteli- und der Ziegler-Kalender (Anm. 47), S. 13-15.

$51 \mathrm{Zu}$ den Reaktionen in der freisinnigen Presse vg!. Wälchli: Martin Disteli 1802-1844 (Anm. 47) passim; sowie Leitess, Noseda u. Wiebel: Martin Disteli 1802-1844 (Anm. 48), S. 87-90.

52 Vgl. dazu Tschopp: Predigen, gefaßt in Lebenssprache (Anm. 1), S. 119-123.

53 Vgl. etwa den Brief vom 8 . Oktober 1844, in dem Carl Bitzius auf einen in Schweizerischen Beobachter erschienenen Artikel reagiert (EB 4, S. 108-113, hier S. 112f.) oder denjenigen vom 22. Februar 1845 (EB 4. S. 170-172). 
Wer kennt nicht den hochverdienten Hrm. Pfarrer Bitzius, sonst auch unter dem Namen ,Jeremias Gotthelf' bekannt, und wer wollte wohl an seiner Tauglichkeit zum akademischen Lehramt zweifeln? Man lese nur den von ihm verfaßten „Neuen Berner-Kalender“ für das Jahr 1845 und die von ihm herrührenden Artikel im „Bernervolksfreund“, die leicht an der körmigen Sprache und den Kraftausdrücken „Sauniggel, Helvötzler, Ohnehosen“ u. s. w., mit welchen er seine politischen Gegner beehrt, zu erkennen sind, nach, und jeder etwaige Zweifel wird schwinden. Der Bernerkalender zeigt namentlich unsern Seelenhirten im schönsten Lichte. Als ächt christlicher Eiferer möchte er besonders das mittelalterliche Kriminalwesen wieder haben: Wahnsinn gilt ihm nicht als Entschuldigung bei Verbrechen, „Kopf ab, das heilte den Wahnsinn gründlich und hemmt eben so gründlich die Ansteckung." (Seite 31) Er zeigt sich höchst entrüstet darüber, daß unsere Strafgesetzgebung die Prügel abgeschafft hat und ihn überdieß noch um den Genuß bringt, alljährlich ein par [!] Kindesmörderinnen unter dem Henkerbeile verbluten zu sehen;*) er folgert daraus, es schiene, der Große Rath habe zuweilen einen Rausch und stellt an denselben die Frage, was ihm lieber sei, eine Hure oder ein Kind (Seite 30). Ferner berichtet er, der Große Rath sei im Dezember 1843 „vor den Pintenwirthen zu dem Kreuze gekrochen," und macht demselben das schöne Kompliment: das Bernervolk wisse am Ende nicht, wo es mit seinem Respekte hin solle; ihn etwa sammt einer Windbüchse und einem Brennhafen wegen Mangel an Platz feil bieten oder auf die Schelme übertragen, welche auf dem Rathhause so ungenirt thun, als wären sie daheim (Seite 39). Aehnliche Lobreden auf die Behörden könnten noch mehrere angeführt werden, wir wollen es aber bei diesen kurzen Bemerkungen bewenden lassen. Dagegen nimmt sich der protestantische Hr. Pfarrer der aufrührerischen Klöster im Aargau mit einer rührenden Zärtlichkeit an; der Aargauerregierung wirft er lange Finger und Diebsgelüste vor (Seite 31) und ruft sogar des Himmels Fluch auf dieselbe herab, weil sie es wagte, jenen Meuterern das Handwerk zu legen [...]. Noch schlechter kommen die armen Walliser Liberalen weg: „Sauniggel und Buben" sind sie sammt und sonders, - und mit dem Titel "Jagdhunde“ sollen sie sich noch besonders geehrt fühlen. (Seite 32). Daß sich die Tessiner Regierung ebenso wenig der Gnade des Hrn. Pfarrers erfreuen kann, versteht sich von selbst, gehört sie doch auch zu den verdammten Liberalen: es wurde dort im April „gestochen, gelogen und gewaltig despötelet;" die Tessiner sind nicht „F reigewordene“ sondern „Freigelassene“. (Seite 32). Ja sie sind sogar schuld - an dem schlechten Wetter im Juli. (Seite 34). Der Papst hat sich hingegen wieder des besondern Wohlwollens des Kalendermachenden Hm. Pfarrers zu erfreuen; der russische Kaiser wird hart angelassen, daß er es gewagt hat, mit dem Papste zusammenzubeißen. (Sapperment! der wird Angst bekommen, wenn er hört, daß Hr. Bitzi gegen ihn auftritt!) [...] $\mathrm{Daß}$ ferner das Sittliche und Aesthetische in dem Kalender würdig repräsentirt ist, das beweisen z. B. die schönen Auszüge „aus Lisebethlis Stammbuch“, aus welchen wir zur Probe bloß die Stelle hervorheben: „wenn ich nur d's Geld wieder hätt, z' Mensch kónnt sy wo nehs wett"! (Seite 67 u. f.) und die bildliche Darstellung zweier kotzender Bauem (Seite 72). Eben so sehr macht sich derselbe um die Volksaufklärung verdient; auf Seite 33 u. 34 z. B. wird den atheistischen Gelehrten tüchtig über's Maul gefahren, daß sie es wagen zu erklären, ein Komet sei eine Naturerscheinung wie jede andere und - keine Zomruthe Gottes, wie man in den gottseligen Zeiten des Mittelalters glaubte; diesen beseligenden Aberglauben will Hr. Bitzius im Volke erhalten wissen! [...]

*) Diese Zärtlichkeit ist ganz im Sinne der Worte des Verf. Seite 4I: „Es ist die Sanftmuth. welche den Sünder nicht todt schlägt, ihn nicht verflucht" u. s. w. ${ }^{54}$

${ }^{54}$ Schweizerischer Beobachter 12. Jg. Nr. 121 vom 8. Oktober 1844 , S. $487 f$. 
Der Widerstand, der Gotthelfs Neuem Berner-Kalender erwuchs, hängt nicht in erster Linie damit zusammen, daß er das Modell des volksaufklärerischen Kalenders nur partiell realisierte und daß er politisch Stellung bezog. Wie das Echo, auf das der Schweizerische Bilderkalender stieß, belegt, fanden gerade in den $1840 \mathrm{er}$ Jahren auch jene Kalender ihr Publikum, die mit Vehemenz in politische Debatten eingriffen. Mit seinen Anleihen sowohl beim aufgeklärten als auch beim religiösen und vor allem politischen Kalender handelte es sich demnach bei Gotthelfs Neuem Berner-Kalender um eine Publikation, die - so könnte man meinen - unterschiedliche Leserbedürfnisse zu befriedigen vermochte und deshalb besondere Verbreitung hätte finden müssen. Wenn dem nicht so war, ist dies wesentlich dem Umstand geschuldet, daß die eigenwillige politische Haltung des Pfarrers von Lützelflüh kaum das Attribut ,mehrheitsfähig' verdient. Wie er in einem Brief an den befreundeten Theologen Karl Rudolf Hagenbach schreibt, verstand er sich weiterhin als, wenn auch skeptischer, Freisinniger und dürte deshalb im katholisch-konservativen Lager nicht nur wegen seiner Konfessionszugehörigkeit bestenfalls verhaltene Zustimmung gefunden haben. ${ }^{55} \mathrm{Zu}$ gleich jedoch übte er entschiedene Kritik am politischen Radikalismus und vertrat Positionen, die ihn als Konservativen erscheinen lassen mußten. ${ }^{56} \mathrm{Da}$ er sich damit nicht nur in seinem Heimatkanton in der Minderheit befand, belegt nicht zuletzt die Tatsache, daß es schließlich die liberalen Kräfte waren, die sich 1848 im Sonderbundskrieg durchsetzten und die politischen Strukturen der daraus hervorgehenden Confoederatio helvetica bestimmten. Unangemessen waren in der Perzeption der mehrheitlich mit liberalen Postulaten sympathisierenden Zeitgenossen, so das Fazit, weniger die spezifische Gestalt des Neuen Berner-Kalenders und der darin vorherrschende satirisch-polemische Ton als vielmehr die zunehmend als anachronistisch empfundene konservative Tendenz seines Verfassers.

Aus dem offenkundig politischen Charakter des Neuen Berner-Kalenders ergibt sich die Notwendigkeit, ihn im Kontext der weiteren publizistischen Tätigkeit Gotthelfs zu betrachten. Während der Status des Neuen Berner-Kalenders innerhalb des dichterischen Gesamtwerks Gotthelfs bereits früh ins Blickfeld der Germanisten rückte, ${ }^{57}$ sind die Bezüge zwischen dem Kalenderschaffen des Emmentaler Pfarrers und seiner journalistischer Tätigkeit noch kaum thematisiert worden. Damit ist ein Desiderat der Forschung benannt, das in meinen abschließenden Darlegungen nicht mehr ausgeführt, sondern nurmehr skizziert werden kann. In die Jahre 1831 bis 1845 fallen nicht nur ein erheblicher Teil der dichterischen Produktion Gotthelfs und die Beiträge für den Neuen Berner-Kalender, sondern auch dessen freie Mitarbeit beim Berner Volksfreund, einem von den Brüdern Johann Ludwig und Karl Schnell in Burgdorf

55 Er habe. so Gotthelf am 5. Oktober 1841 an Hagenbach, sich „von Anfang entschieden unter die freisinnige Fahne gestellt und stehe noch darunter. Das gibt mir nicht nur das Recht, sondern macht es mir entschieden zur Pflicht," fährt er fort, ,gegen alle MiBbräuche der Freiheit, gegen alle, die von der Freiheit schmarotzen wollen, unumwunden derb und hart zu reden" (EB 5, S. 159).

56 Eine differenzierte Betrachtung von Gotthelfs politischer Position findet sich bei Hellmut Thomke: Gotthelfs .Konservativismus' im europāischen Kontext. In: Erzählkunst und Volkserziehung (Anm. 1), S. 227-241.

57 Vgl. Itten: Gotthelf als Kalenderschreiber (Anm. 11), S. 23-25. Ein systematischer Vergleich zwischen Gotthelfs literarischem Erzählwerk und dessen Kalenderbeitrăgen steht alterdings noch aus. 
gegründeten liberalen Organ. ${ }^{58}$ Es überrascht nicht, daß sich zwischen dem Neuen Berner-Kalender und den im Berner Volksfreund erschienenen Artikeln eine Reihe von Überschneidungen ergeben, die den engen Zusammenhang nicht nur zwischen dem poetischen und dem publizistischen Schaffen Gotthelfs, sondern auch zwischen seiner Kalenderredaktion und dessen Zeitungsmitarbeit belegen. So findet sich im Berner Volksfreund vom 5. Januar 1840 ein Artikel „Über Ein- und Ausfuhr, Flachsanbau und Spinnerei“, der im Sinne einer protektionistischen Wirtschaftspolitik die Problematik der Einfuhr von englischem Flachsgarn erläutert und in diesem Kontext die in Burgdorf bevorstehende Errichtung einer Spinnerei durch die Gebrüder Miescher hervorhebt. ${ }^{j 4}$ Letztere wiederum bildet den Ausgangspunkt in „Marei, die Kuderspinnerin, und ihr Tröster", einem im Neuen Berner-Kalender auf das Jahr 184l erschienenen Dialog zwischen einer alten Frau, die durch die entstehende Spinnerei ihren geringen Ertrag aus der Garnproduktion bedroht sieht, und einem anonymen Ich-Erzähler, der Marei davon zu überzeugen versucht, daß die schweizerische Textilindustrie auf Spinnereien angewiesen sei und letztlich auch die vor allem für den Eigenbedarf spinnenden Heimarbeiterinnen aus der industriellen Garnproduktion Nutzen zögen. ${ }^{60}$ Auch der im Berner Volksfreund vom 8. Juli 1841 erschienene „Bericht über das Kantonalschießen in Sumiswald"61 findet seinen Widerhall im Neuen Berner-Kalender. Während „Etwas vom Sumiswalder Schießet ${ }^{\text {“62 }}$ mit freundlicher Ironie das Verhalten eines vornehmen Engländers, der am Schützenfest teilnimmt, beschreibt, konzentriert sich der Zeitungsartikel auf das harmonische Treiben der Festgesellschaft und - auf einen von Gotthelf persönlich vorgetragenen Trinkspruch, der in voller Länge zitiert wird.

Es würde zu weit führen, die Berührungspunkte zwischen dem Neuen BernerKalender und Gotthelfs Artikeln im Berner Volksfreund im Einzelnen zu benennen. Auch eine Analyse der medienbedingten Unterschiede in der Behandlung desselben Themas würde den Rahmen dieses Beitrags sprengen. Meine knappen Hinweise mögen genügen, um deutlich werden zu lassen, mit welchen Herausforderungen sich eine kommentierte Edition der publizistischen Arbeiten Gotthelfs konfrontiert sieht: Eine adäquate Einschätzung des Neuen Berner-Kalenders ist nur dann möglich, wenn man ihn in jene publizistischen Kontexte stellt, die seine (nicht nur) zeitgenössische Relevanz determinieren. Bedeutsam ist zum einen das dem Neuen Berner-Kalender zugrunde liegende Gattungsverständnis, das dazu beiträgt, die Zielsetzungen seines Herausgebers zu erbellen. Nicht weniger signifikant ist die Berücksichtigung jener zeitgleich erscheinenden Kalender, auf die der Neue Berner-Kalender implizit oder explizit reagiert. Die in der Forschung wiederholt formulierte These der Singularität

58 Die im Berner Vorksfreund erschienenen Beitrüge Gothelfs sind abgedruckt in Jeremias Gotthelf: Sămtliche Werke in 24 Bānden. Hrsg. von Rudolf Hunziker u. Hans Bloesch. Dreizehnter Ergänzungsband. Bearb. von Frizz Huber-Renfer: Politische Schriften. Erster Teil. Erlenbach-Zürich 1956 [im folgenden: EB 13] und in Jeremias Gotthelf: Sămtliche Werke in 24 Bänden. Hrsg. von Rudolf Hunziker u. Hans Bloesch. Vierzehnter Ergânzungsband. Bearb. von Fritz Huber-Renfer u. Kun Guggisberg: Politische Schriften. Zweiter Teil. Erlenbach-Zürich 1966.

59 Vgl. EB 13.S. 162-166.

60 Vgl. SW XXIII, S. 181-195.

61 Vgl. EB 13,S. 209-211.

62 Vgl. SW XXIII, S. 405-409. 
von Gotthelfs Kalender erfährt dadurch, wie ich zu zeigen versucht habe, eine Relativierung; der Neue Berner-Kalender erscheint vor dem Hintergrund der politischen und medialen Entwicklungen um 1840 zumindest hinsichtlich seiner inhaltlichen Konzeption und seiner rhetorischen Gestaltung als durchaus zeitgemäß. Von Belang ist schließlich und drittens Gotthelfs journalistisches Wirken, das in engem Zusammenhang mit seinem weiteren publizistischen Schaffen zu sehen ist. Gotthelf hat seine umfangreiche und vielgestaltige schriftstellerische Tätigkeit immer als ein Ganzes verstanden, hat seinen literarischen Erzählungen und Romanen, seinen Predigten und seinen publizistischen Arbeiten den gleichen hohen Stellenwert beigemessen. Nur wer aus dieser Einsicht die Konsequenzen zieht und die Textproduktion Gotthelfs in ihrer Gesamtheit in den Blick nimmt, wird sie als jenes vernetzte System beschreiben können, als das sie von ihrem Autor von Anfang an intendiert war. 